\title{
Blood safety: new challenges \& opportunities in developing nation
}

Keywords: blood transfusion, HIV, hepatitis b, syphilis, HCV, HBV

\section{Introduction}

In a country with limited resources and where cost economics playing vital role we have to delicately strike a balance between cost, quality, manpower \& infrastructural constraints as well as opening for new testing technologies prevalent in the world. In order to keep pace with the growth and safety for blood receipts we need to gear up for acceptance and incorporation latest testing facilities for our blood bank. ${ }^{1}$

Blood transfusion is an essential and life-saving support within the health care system but the safety and availability of blood and transfusion is not assured. The threats include: - (a) an insufficient number of voluntary non-remunerated repeat regular blood donors to ensure an adequate supply of safe blood and blood components. (b) Risk of the transmission of infections such as HIV, hepatitis B and $\mathrm{C}$, and syphilis through unsafe transfusion. (c) Unnecessary transfusions, which needlessly expose patients to the risk of acute or delayed reactions and transfusion-transmitted infections. (d) Errors in the transfusion of blood and blood components. ${ }^{2}$

Blood safety depends on the following factors :- (a) The incidence and prevalence of transfusion - transmissible infections in the blood donor population. (b) The effectiveness of the blood donor education and recruitment programme and procedures for donor selection and screening, including the deferral or exclusion of unsuitable donors. (c) The quality of screening of all donated blood for transfusion transmissible infections. (d) The quality of blood grouping, compatibility testing, component preparation and the storage and transportation of blood products. (e) The extent to which blood and blood products are prescribed only when there is no alternative to transfusion for the particular patient. (f) The reliability of the system for ensuring that patients receive blood that is compatible with their blood group, red cell antibodies and other special requirements. ${ }^{2}$

Safe blood is the one which does not cause any adverse event in the form of infection or reaction when transfused to the recipient. Blood safety has gained importance in the recent years especially after introduction of HIV infection in the list of Blood transmissible infections. Blood safety in the developed counties has considerably improved especially for transmission of viral infections, as the possibility of getting transfusion transmitted HIV, HCV and HBV is to the tune of 1 in 2million, 1 in 1.5 million and 1 in 3lakhs respectively, but the improvement in the developing world including India has not kept pace with the western world. ${ }^{3}$

Blood safety is a highly complex and multi-pronged problem for which here are no easy solutions. It is imperative, that the decision makers understand the factors governing the availability of safe blood and take timely and meaningful decisions to provide a safe blood transfusion. Tremendous focus on blood safety and overall transfusion safety depends upon the coordinated linkages of many processes from donor collection to transfusion. ${ }^{4}$
Volume 4 Issue 5 - 2017

Subhashish Das
Department of Pathology, Sri Devaraj Urs Medical College, India

Correspondence: Subhashish Das, Professor of Pathology, Sri Devaraj Urs Medical College, Sri Devaraj Urs Academy of Higher Education and Research, Tamaka, Kolar, Karnataka-56310I, India, Email daspathology@gmail.com

Received: February 28, 2017| Published: May 02, 2017

Blood safety does not simply mean blood that is free from TTI; it may be even related to ensuring the right blood grouping. It would be pertinent here to say "safety' means right quality, which comes through a comprehensive system encompassing every step, starting from donor selection to transfusion to the patient. Therefore, it is a continuous process comprise of various steps. The challenge before us is how to do it and ensure it is practiced on an ongoing basis. Therefore, we need a system of ensuring quality blood or minimizing the risk to the recipient or blood safety which can uniformly implemented in the blood banks /centres and includes all steps followed for collection processing. ${ }^{4}$

However, even though screening techniques are a very reliable way to detect many lethal viruses, blood transfusion still poses risks for the following reasons: i) the window period of pathogens during which an infectious donor cannot be detected has been reduced, but not eliminated by NAT screening because NAT testing is generally carried out on pooled donor samples which can raise the chance of infection due to dilution of the signal from infected donors; ii) new emerging pathogens may enter the blood supply; iii) parasites and bacteria also represent an infective risk. Routine serological testing currently does not test for various parasitic diseases, and limited test methods exist. ${ }^{5}$

Bacteria may be present in asymptomatic donors or, more frequently, enter the blood during collection. Efforts to reduce the risk of bacterial contamination of platelet products is of high importance since platelets are stored at room temperature, allowing for bacterial proliferation, and also because platelets are frequently given to patients with impaired immune systems who are more susceptible to bacterial infections. In developing countries the risk of all transfusiontransmitted infections is still high due to insufficient funding and organization of health services.

Bacterial contamination, one of the earliest recognized complications of stored blood, remains an important cause of transfusion-related mortality and morbidity. Bacteria are believed to originate with the donors, either from the venipuncture sites or from unsuspected bacteremia. RBCs are primarily infected with psychrophilic gram-negative organisms such as Yersinia enterocolitica and Serratia liquifaciens. Other pathogens such as staphylococcus, klebsiella, Enterobacter, E coli, streptococcus, 
Bacillus and Pseudomonas are also known to infect erythrocytes. Discarding the first aliquot of donor blood removed has been proposed as a measure to reduce bacterial contamination of blood components based on association of skin bacteria that enter at time of phlebotomy. Various approaches to detect bacterial contamination besides visual inspection before release of the component are Gram's stain and culture, endotoxin assays and detection of bacterial nucleic acids by amplification techniques. $^{5}$

Days are gone when patients was not aware of his rights but now in the era of consumer led world, patients are well versed with their rights for the services they are entitled for. It is therefore, our responsibility to ensure that blood or its products going to be used must confirm to all its quality and safety parameters. In order to provide desired service to their patients and also to avoid certain litigations, blood bank/Centre must apply a systematic approach which can easily be achieved by maintaining highest degree of safety standards. ${ }^{5}$

It is therefore important that the quality and safety of all blood and blood products be assured throughout the process from the selection of blood donors to the administration of the product to the patient. ${ }^{5}$

There are many definitions of quality, one of the simplest and most appropriate being "fit for purpose". In the context of blood transfusion, this means the setting and meeting of basic national quality standards and then continual quality improvement to ensure the safety of the transfusion process.

Keeping this in view, the following steps are being suggested to improve blood safety throughout the world including the poor and resource constraint developing nation.

\section{Nucleic acid testing (NAT)}

Nucleic acid Testing (NAT) is a method of testing blood that is more sensitive than conventional tests that require the presence of antibodies to trigger a positive test result. While an infection occurs, NAT is used to detect the low levels of viral genetic material present in the body.

The advantages of NAT include: (a) Detects low levels of viral RNS or DNA. (b) Highly sensitive and specific for viral nucleic acids. (c) Provides additional layer of safety to the blood supply. (d) Detects infection earlier than other screening methods, narrowing the window period. (e) Ability to detect viral mutant and occult infections. ${ }^{6}$

\section{Pathogen inactivation}

The ultimate goal of pathogen inactivation is to maximally reduce the transmission of potential pathogens without significantly compromising the therapeutic efficacy of the cellular and protein constituents of blood. This must be accomplished without introducing toxicities into the blood supply and without causing neo antigen formation and subsequent antibody production. ${ }^{6}$

\section{Molecular blood grouping}

High-throughput genotyping platforms that utilize microarray and chip technologies have now been developed. It is now possible to predict blood group phenotypes from tests on genomic DNA, with a high degree of accuracy. Molecular immuno hematology is changing practice in blood centers, hospital blood banks, and reference laboratories. ${ }^{6}$

\section{Technological innovation for transfusion safety}

Radiofrequency-based systems are on the near horizon. Nearly all systems begin with the application of machine-readable data to the patient's wristband. The third error spot- the decision to transfuse-will be a challenging area for future application of information technology. Computerized physician order-entry, decision support and ultimately active computer-based decision-making are expected to enhance transfusion decisions. Healthcare workers, administrations, and regulators need to embrace the used of new technology in order to reduce errors and improve safety for patients. ${ }^{6}$

\section{Evidence based transfusion guidelines}

Will-developed, high-quality practice guidelines should be valid, reliable, reproducible, and clinically applicable statements developed using the principles of evidence-based medicine (EMB). The first principle of EBM is that practice guidelines should be based on thorough, unbiased, and critically evaluated systematic review of the applicable medical literature. The second principle is that evidence is necessary, but not sufficient, for decision making and that an assessment of the quality of the evidence should be issued separately from the guidelines. When developing such guidelines it will be important to ensure that the guidelines address the questions that prescribing clinicians might ask. ${ }^{6}$

\section{Platelet additive solutions}

The existing shelf lives of PCs are for 5 days and thereafter a progressive loss of platelet viability and function occur during platelet storage. Platelet storage remains a major challenge to transfusion services. Technologies and strategies are desired to allow extended storage without causing an unacceptable loss of product quality.

Platelets stored in PAS have been demonstrated to have a lower risk for allergic transfusion reactions and also result in a lower incidence of other plasma -associated transfusion reactions, such as ABO hemolytic reactions and transfusion-related acute lung injury (TRALI).

\section{Conclusion}

The aim of quality in blood transfusion services (BTS) is to ensure the provision of safe transfusion of blood and its components. Blood bank is the only laboratory directly responsible for the patients with no intervening physician (doctor) interpretation, so any mistake in blood transfusion service (blood banks) can be disastrous. In order to prevent mistakes in blood transfusion services it is essential to have adequate quality assurance in blood transfusion services. There are many terms used in relation to quality i.e. quality control, quality assurance and quality audit. ${ }^{7}$

However, in our rural and resource constrained set up blood banks operate in total isolation; their standards vary from states to states, cities to cities, and from one centre to another centre in the same city. The hospital based decentralized blood banking system has led to a skewed distribution of resources and makes difficult any implementation of a stringent quality control program.

Recent concept of blood transfusion emphasizes on the transfusion of component therapies instead of transfusion of whole blood. Throughout the world blood services aim to provide a lifesaving service by ensuring an adequate supply of safe blood. The approach towards blood transfusion practices should be based on "Four Principles" of autonomy, beneficence, non-malfeasance, and justice which offers a common, basic moral analytical framework and a common, basic moral language to think about ethical issues in any field of health care services. 
The practice of transfusion medicine involves a number of ethical issues because blood comes from human beings and is a precious resource with a limited shelf life. It involves a moral responsibility of the physicians and blood banks towards both the donors and the patients. Decisions must be based on four principles as mentioned earlier and of course, the Hippocrates' principles of "Primum non nocere" (i.e. first do no harm).

Today's transfusion medicine is sophisticated and complex involving an understanding of a variety of scientific disciplines in addition to the medical management of patients. Transfusion medicine seems to be on the threshold of several major changes which will add to the complexities of transfusion medicine and would continue to provide exciting opportunities for transfusion medicine physicians, scientists, and technologists in the future. Haemovigilance systems would help determine trends of TTIs and the need to implement screening for the newer infections. Over the years, there has been a substantial decline in the incidence in the transfusion-transmitted infection due to improvement in donor screening, testing and viral inactivation of blood products, particularly in developed nations. However, in developing nations, blood safety continues to be a major problem due to high prevalence of infections markers among blood donors compounded with the problem of limited resources that preclude the use of sophisticated, sensitive but expensive technologies for screening of blood products. The last two decades have also witnessed surfacing of new and re-emerging infections. Hence, despite stringent donor eligibility criteria, improved donor screening and introduction of sophisticated technology, transfusion-transmitted infection continues as a challenge for transfusion experts.

\section{Acknowledgements}

None.

\section{Conflict of interest}

The author declares no conflict of interest.

\section{References}

1. Bray TJ, Salil P, Weiss HA, et al. Intervention to promote appropriate blood use in India. Transfus Med. 2002;12(6):357-366.

2. Wake DJ, Cutting WA. Blood transfusion in developing countries: Problems, priorities and practicalities. Trop Doct. 1998;28(1):4-8.

3. Gibbs WN, Corcoran P. Blood safety in developing countries. Vox Sang. 1994;67(4):377-381.

4. Jolly JG. Deplorable state of blood transfusion services in India. J Indian Med Assoc. 2007;105(9):491.

5. Sazama K. Transfusion medicine for the practicing pathologist/physician with responsibilities for the transfusion service. Arch Pathol Lab Med. 2007;131:688-689.

6. Harmening DM. Modern blood banking and transfusion practices. 5th ed. USA: FA Davis Co.; 2005.

7. Macpherson CR, Domen RE, Perlin TM. Ethical issues in transfusion medicine. India: AABB Press; 2001. 